\title{
A Real-World, Prospective, Non-interventional Study of Adults with T2D Switching to IDegAsp from Glargine U100 or U300 in Japan
}

\author{
Fumika Shigiyama · Lei Liu · Helene Nordahl · Ryo Suzuki • \\ Yuiko Yamamoto · Takahisa Hirose
}

Received: April 15, 2021 / Accepted: July 7, 2021 / Published online: July 25, 2021

(C) The Author(s) 2021

\begin{abstract}
Introduction: This real-world study investigated glycaemic control and quality of life (QoL) in insulin-experienced Japanese patients with type 2 diabetes (T2D) who switched to insulin degludec/insulin aspart (IDegAsp).

Methods: This was a prospective, non-interventional, open-label, single-arm study. Eligible patients were adults (aged $\geq 20$ years) with T2D, previously treated with insulin glargine 100 or 300 units/mL (glargine U100/U300) with or without prandial insulin, who switched to IDegAsp as part of routine practice. Change from baseline to end of study (EOS; 26 weeks after initiation or IDegAsp discontinuation) in
\end{abstract}

Supplementary Information The online version contains supplementary material available at https:// doi.org/10.1007/s13300-021-01117-8.

F. Shigiyama $\cdot$ T. Hirose $(\bowtie)$

Division of Diabetes, Metabolism and

Endocrinology, Department of Medicine, Toho

University Graduate School of Medicine, 6-11-1

Omori-Nishi, Ota-ku, Tokyo 143-8541, Japan

e-mail: takahisa.hirose@med.toho-u.ac.jp

L. Liu · H. Nordahl

Novo Nordisk A/S, Søborg, Denmark

R. Suzuki

Tokyo Medical University, Tokyo, Japan

Y. Yamamoto

Novo Nordisk Pharma Ltd., Tokyo, Japan the following endpoints was assessed by adjusted mixed models for repeated measures: glycated haemoglobin (HbA1c; primary endpoint), fasting plasma glucose (FPG), insulin dose and total Diabetes Therapy-Related Quality of Life (DTR-QoL) score. Non-severe hypoglycaemia was assessed in the 4-week period prior to initiating IDegAsp and in the 4 -week period before EOS or discontinuation using negative binomial regression.

Results: The full analysis set included 236 patients from 29 centres in Japan with mean $( \pm \mathrm{SD})$ age 63.2 years ( \pm 12.3$)$, HbA1c $7.7 \%$ $( \pm 1.0)$ and diabetes duration $14.9( \pm 9.3)$ years. After 26 weeks with IDegAsp, HbA1c (estimated change $-0.1 \%[-0.2 ; 0.0]_{95} \%$ confidence interval $(\mathrm{CI})$, $p=0.3036) \quad$ and $\quad$ FPG $\quad(-7.5 \mathrm{mg} / \mathrm{dL}$ $\left.[-23.5 ; 8.5]_{95 \% \mathrm{CI}} p=0.3477\right)$ were maintained; there were significant reductions in basal and total insulin dose: estimated change of -3.4 units/day $[-3.8 ;-3.0]_{95 \%}$ CI and -1.0 units/day $[-1.9 ;-0.1]_{95 \% \text { CI}}$, respectively (both $p<0.05$ ). Non-severe hypoglycaemia rates were similar in the periods before and after initiating IDegAsp, while there was a significant improvement in total DTR-QoL score after 26 weeks with IDegAsp $(p=0.0012)$.

Conclusion: These real-world data suggest that switching to IDegAsp from glargine U100 or U300 was well tolerated in a Japanese population with T2D, with no new safety or tolerability signals, and associated with maintenance of glycaemic control and improved QoL. 
Trial registration: This study is registered at ClinicalTrials.gov: NCT03745157.

Keywords: Insulin degludec/insulin aspart; Japanese population; Quality of life, real-world evidence; Type 2 diabetes

\section{Key Summary Points}

Why carry out this study?

Clinical inertia-the failure to establish appropriate targets and escalate treatment to achieve treatment goals-is prevalent in clinical practice, particularly when considering the initiation of insulin in patients with type 2 diabetes (T2D).

Randomised controlled trials (RCTs) of insulin degludec/insulin aspart (IDegAsp), a co-formulation of basal and prandial insulin delivered by a pre-filled injection pen either once or twice daily, have demonstrated efficacy and safety in insulin-naïve and insulin-experienced Japanese patients with T2D.

However, there are currently no RCTs evaluating the switch to IDegAsp from insulin glargine in Japanese patients with T2D, and real-world data are lacking.

The present real-world, prospective, noninterventional, single-arm study investigated glycaemic control and quality of life (QoL) in patients who switched to IDegAsp from insulin glargine 100 or 300 units/mL, with or without prandial insulin and as part of routine clinical practice, in a Japanese population with T2D.

\section{What was learned from the study?}

Switching to IDegAsp for 26 weeks was associated with maintenance of glycaemic control, a lower daily total and basal insulin dose requirement, a similar incidence of non-severe hypoglycaemia and improvements in QoL in comparison with baseline prior to the switch.
These real-world data are in alignment with the findings of previous RCTs-that initiating IDegAsp in insulin-experienced Japanese patients with T2D is well tolerated and associated with the maintenance of glycaemic control-and may provide insights on switching from insulin glargine to IDegAsp.

\section{INTRODUCTION}

In 2019, the overall prevalence of diabetes in Japan was $7.9 \%$, with 7.4 million Japanese adults living with the disease [1]; this is predicted to rise to 9.7 million (9.8\% prevalence) by 2030 , mostly due to population ageing [2]. Current Japan Diabetes Society (JDS) clinical guidelines recommend an oral blood glucose (BG)-lowering medication and/or a glucagonlike peptide-1 receptor agonist (GLP-1 RA) in patients with type 2 diabetes (T2D) who have not achieved individualised treatment targets within 2-3 months of lifestyle changes [3]. Guidelines recommend increasing the dose of or adding further oral BG-lowering medications, or adding a GLP-1 RA/insulin to the therapeutic regimen of patients who do not achieve their glycaemic goals with their current intervention [3]. Intensive insulin therapy is recommended in patients who still do not achieve their glycaemic goals with the previous interventions [3].

Japanese patients with T2D are characterised by reduced insulin secretion compared with Caucasians $[4,5]$, while a traditionally carbohydrate-rich diet [6] emphasises the need to effectively target prandial hyperglycaemia. Due to these pathophysiologic characteristics associated with Japanese patients, and the progressive nature of T2D, initiation and intensification of insulin therapy is often required [7]. Clinical inertia-the failure to establish appropriate targets and escalate treatment to achieve treatment goals-is prevalent in clinical practice, particularly when considering the initiation of insulin in patients with T2D [8]. The reasons for clinical inertia in 
insulin initiation are multifactorial and include preconceptions regarding the increased burden of multiple injections and BG monitoring, as well as the complexity of multi-medication regimens. However, inertia is responsible for substantial preventable diabetes-related complications and associated healthcare costs [8].

Insulin degludec/insulin aspart (IDegAsp) is a soluble co-formulation of insulin degludec consisting of a basal insulin analogue with an ultra-long duration of action and insulin aspart, a rapid-acting prandial insulin analogue, in a single injection [9]. The individual components of IDegAsp maintain their distinct pharmacokinetic and pharmacodynamic properties in IDegAsp, thereby avoiding the 'shoulder effect' associated with pre-mixed insulins. This effect may increase the risk of hypoglycaemia as the long-acting basal component can protract the action of the rapid-acting prandial component [10]. IDegAsp is delivered subcutaneously via a pre-filled insulin pen, thus eliminating the need for re-suspension [9]. It is administered once or twice daily [9], with the potential to reduce the daily number of insulin injections when compared with basal-bolus insulin regimens [10].

Treat-to-target randomised controlled trials (RCTs) of IDegAsp have demonstrated similar efficacy to various insulin comparators in global patient populations across the clinical continuum of T2D, including in insulin-experienced patients [11-14]. Similar efficacy with a good safety and tolerability profile has been demonstrated in RCTs in Japan of both once-daily IDegAsp versus insulin glargine (glargine) in insulin-naïve patients and twice-daily IDegAsp versus biphasic insulin aspart 30 (BIAsp 30) in insulin-experienced patients [15-17]. However, there are currently no RCTs evaluating the switch from insulin glargine to IDegAsp in Japanese patients with T2D.

Earlier studies noted improvements with IDegAsp versus comparators in terms of glycaemic control endpoints in Japanese patients with T2D [15-17]. In 2015, these results supported the launch of IDegAsp in Japan for patients with T2D requiring insulin therapy. While RCTs are considered the 'gold standard' in research, the rigorous design and strict eligibility criteria of most RCTs mean that their results may not be generalisable to general patient populations [18]. RCT data can be complemented by real-world studies, which provide a bridge from the results of RCTs to daily clinical practice [18].

The present real-world study was undertaken to investigate glycaemic control and quality of life (QoL) in Japanese patients with T2D who switched to IDegAsp from insulin glargine 100 units/mL (glargine U100) or 300 units $/ \mathrm{mL}$ (glargine U300), with or without prandial insulin and as part of routine clinical practice.

\section{METHODS}

\section{Study Design}

This was a prospective, non-interventional, open-label, single-arm study which took place between November 2018 and October 2019 at 29 centres across Japan. The study design is presented in Electronic Supplementary Material (ESM) Fig. S1. The study was conducted in accordance with the Declaration of Helsinki, the Guidelines for Good Pharmacoepidemiology Practice and the Guideline on Good Pharmacovigilance Practices (Module VI) [19-21]. Additionally, the Ethical Guidelines for Medical and Health Research Involving Human Subjects [22] were followed, as required in Japan. The study protocol was approved by the appropriate independent ethics committees/institutional review boards at each site. A full list of the research ethics boards/institutional review boards, with their reference numbers, is provided in ESM Table S1. All patients provided written informed consent prior to participation.

\section{Study Population}

Eligible patients were adults aged $\geq 20$ years who had been diagnosed with T2D for $\geq 26$ weeks prior to signing an informed consent form; who had been treated with a glargine product (either U100 or U300, including biosimilar) for $\geq 26$ weeks prior to switching to IDegAsp, as part of routine clinical practice; and who had an available glycated haemoglobin 
(HbA1c) measurement $\leq 12$ weeks prior to this therapeutic switch. Patients treated with glargine U300 prior to switching must have received glargine U300 for $\geq 12$ weeks prior to the switch to IDegAsp and, as such, could have been treated with glargine U100 for the initial 14 weeks of the minimum 26 weeks of treatment with a glargine product. Patients who had previously received treatment with, or with known hypersensitivity to, IDegAsp were excluded from the study. Pregnant patients were also excluded. A full list of the inclusion, exclusion and withdrawal criteria is presented in ESM Table S2. To ensure the inclusion of patients previously treated with glargine U300, the proportion of patients switching from glargine U100 was capped at 80\%.

Patients were switched to IDegAsp at the discretion of the treating physician, as part of routine clinical practice, and in accordance with local guidelines in Japan. The decision to initiate treatment with IDegAsp was made before, and independently from, the decision to include the patient in the study. IDegAsp was administered via a pre-filled insulin pen at the starting dose selected by the treating physician. All subsequent IDegAsp dose adjustments and the initiation, dose adjustment or discontinuation of other BG-lowering medications (including other insulins) were made at the treating physician's discretion. The analytical datasets and observation periods are described in ESM Method 1.

\section{Study Endpoints}

The primary endpoint was change in HbA1c (as measured by a local laboratory) from baseline to end of study (EOS) or IDegAsp discontinuation. Secondary endpoints included change from baseline to EOS in the following endpoints: daily total, basal, and prandial insulin dose; fasting plasma glucose (FPG; as measured by a local laboratory); and Diabetes Therapy-Related QoL (DTR-QoL) score. The DTR-QoL questionnaire assesses the influence of diabetes treatment on health-related QoL. It consists of 29 items from the following domains: 'burden on social activities and daily activities' (13 items); 'anxiety and dissatisfaction with treatment' (eight items); 'hypoglycaemia' (four items); and 'satisfaction with treatment' (four items). The total DTR-QoL score ranges from 0 to 100 , with 100 representing the highest health-related QoL. Two questions related to willingness to continue IDegAsp treatment (yes/no) and preference for IDegAsp over the previous treatment (yes/no) were posed to all patients at EOS or at IDegAsp discontinuation (including patients who had withdrawn from study).

Additional endpoints included self-reported patient recollection of overall (i.e. at any time) or nocturnal hypoglycaemia in the periods before and after initiating IDegAsp. Non-severe hypoglycaemia was defined as an event with symptoms and/or a self-monitored blood glucose value $\leq 70 \mathrm{mg} / \mathrm{dL}$, while severe hypoglycaemia was defined as an event requiring assistance from another person to actively administer carbohydrate or glucagon, or take another corrective action [23]. Nocturnal hypoglycaemic events were categorised based on the patient's answer to the question, 'how many of these occurred between midnight and early morning?'. Data for adverse events (AEs) were also collected.

Baseline was defined as the visit at which IDegAsp was initiated (week 0). For assessment of the HbA1c, FPG and insulin dose endpoints, baseline was defined as the period $\leq 12$ weeks prior to the treatment initiation visit (week 0 ), with the most recent measurement selected when multiple measurements were available. EOS was defined as the first visit within the period between week 24 and week 34 (termed week 26). If multiple measurements were available for an endpoint within this period, the most recent measurement relative to the EOS visit was selected. Data were collected via an electronic clinical record form completed by the treating physician using available data from patients' medical records and paper-based patient-reported outcomes.

\section{Statistical Analyses}

Assuming an estimated mean \pm standard deviation (SD) change in HbA1c of $0.3 \% \pm 1.3$ and a 
study completion rate of $85 \%$, the enrolment of 236 patients in the study was planned to detect this difference at $90 \%$ power. Patient characteristics, DTR-QoL domain scores and AEs were summarised descriptively. Primary analyses were conducted for the full analysis set (FAS) and the on-treatment observation period via crude and adjusted mixed models for repeated measures (MMRMs) for the change from baseline to EOS for the following endpoints: HbA1c (primary endpoint); FPG; and daily total, basal and prandial insulin dose. For the primary endpoint, the crude and adjusted MMRMs had an unstructured covariance matrix, time and time squared as fixed effects and patient and patient $\times$ time as random coefficients. The crude MMRM included baseline HbA1c and time of baseline HbA1c measurement as covariates. The adjusted MMRM included baseline HbA1c, time, age, sex, diabetes duration, body mass index (BMI), baseline prandial insulin, baseline GLP-1 RA and study site as covariates.

Secondary analyses of the primary endpoint included: (1) an intention-to-treat analysis that considered the FAS and the in-study observation period (via crude and adjusted MMRMs, similar to the primary analysis), and (2) crude and adjusted analyses of covariance (ANCOVAs) that considered the complete-on-treatment analysis set. The crude ANCOVA included baseline $\mathrm{HbA} 1 \mathrm{c}$ and time of baseline HbA1c measurement as covariates. The adjusted ANCOVA included the following covariates: baseline HbA1c, age, sex, diabetes duration, BMI, baseline glargine (U100/U300), baseline prandial insulin and baseline GLP-1 RA.

Primary analyses via crude and adjusted MMRMs were repeated for the secondary endpoints (change from baseline in FPG and total, basal and prandial insulin dose). The MMRM structure was similar to those used for the primary endpoint but with baseline HbA1c substituted with the baseline value of the relevant endpoint and inclusion of an additional covariate of baseline sulphonylurea/glinide. Secondary analyses were performed for the change from baseline in daily total, basal, and prandial insulin dose via crude and adjusted ANCOVAs that considered the FAS and the on- treatment observation period. The ANCOVA structure was similar to those used for the primary endpoint but substituted baseline HbA1c with the relevant baseline insulin dose. Due to the small number of patients receiving prandial insulin, the planned analyses of this endpoint could not be performed, and these data were summarised descriptively. The change from baseline in total DTR-QoL score was analysed using a crude MMRM with time (visit) as fixed coefficient and a random intercept to accommodate variability in scores at baseline.

The incidence of hypoglycaemia (overall non-severe, nocturnal non-severe, overall severe and nocturnal severe) was analysed in the FAS for the on-treatment observation period using negative binomial regression models with the log-transformed follow-up time as offset. Models compared the incidence within the 4 weeks prior to initiating IDegAsp with that within the 4 weeks prior to EOS or discontinuation for non-severe hypoglycaemic events, and within the 26 weeks prior to initiating IDegAsp with that within the 26 weeks prior to EOS or discontinuation for severe hypoglycaemic events. Secondary analyses were performed for the complete-on-treatment analysis set using negative binomial regression models. Due to a low incidence of severe hypoglycaemia, planned statistical analyses could not be performed for the overall and nocturnal severe hypoglycaemia endpoints, which were summarised descriptively.

The following post hoc analyses were conducted: the primary analysis of the primary endpoint was repeated for the FAS stratified by age ( $<65$ years and $\geq 65$ years); the secondary analysis of the primary endpoint was repeated for the complete-on-treatment analysis set stratified by baseline HbA1c $(<7.0 \%$; $\geq 7.0$ to $<8.0 \%$; $\geq 8.0 \%$ ); and a crude ANCOVA analysed the change from baseline in total DTRQoL score in the complete-on-treatment analysis set stratified by baseline HbA1c $(<7.0 \% ; 7.0$ to $<8.0 \%$; $\geq 8.0 \%$ ).

Missing data were not imputed, and $p<0.05$ was considered to be statistically significant. The results of the primary analyses of endpoints are presented throughout the results, with the adjusted analysis reported preferentially when 
both crude and adjusted primary analyses were performed. The results of additional analyses are reported in the ESM and referred to in the main text.

\section{RESULTS}

\section{Study Population}

Overall, 246 patients across 29 centres in Japan were enrolled, of whom 236 were eligible and included in the FAS. Ten patients did not meet study inclusion/exclusion criteria and had been enrolled in error; they were withdrawn from the study (ESM Fig. S2). In total, 229 patients (97.0\% of the FAS) completed the study, while 19 patients $(0.1 \%)$ discontinued IDegAsp during the study. The baseline characteristics of the study population are presented in Table 1 . At baseline, patients had mean $\pm \mathrm{SD}$ age of $63.2 \pm 12.3$ years, $\mathrm{HbA} 1 \mathrm{c}$ of $7.7 \pm 1.0 \%$, BMI of $25.8 \pm 4.7 \mathrm{~kg} / \mathrm{m}^{2}$ and diabetes duration of $14.9 \pm 9.3$ years. Biguanides and dipeptidyl peptidase- 4 inhibitors were the most utilised non-insulin BG-lowering medications, used by $94(54.3 \%)$ and $81(46.8 \%)$ patients, respectively. Prior to switching to IDegAsp, most patients $(n=143 ; 60.6 \%)$ were treated with glargine U100 at a mean \pm SD dose of $15.2 \pm 10.3$ units, while the majority $(n=225 ; 95.3 \%)$ of patients switched to receive IDegAsp administered once daily.

\section{Glycaemic Control}

Observed mean \pm SD HbA1c was $7.6 \pm 1.0 \%$ after 26 weeks of treatment with IDegAsp and $7.7 \pm 1.0 \%$ at baseline (estimated change $-0.1 \% \quad[-0.2 ; 0.0]_{95 \%}$ confidence interval (CI), $p=0.3036$ ) (Fig. 1). Results of the crude and secondary analyses were consistent with the main result: HbA1c was similar at baseline and after 26 weeks' treatment with IDegAsp ( $p \geq 0.05$ for all) (ESM Table S3). Similarly, FPG was maintained over 26 weeks of treatment with IDegAsp (EOS $148.7 \mathrm{mg} / \mathrm{dL}$; baseline $140.4 \mathrm{mg} / \mathrm{dL}$; estimated change $-7.5 \mathrm{mg} / \mathrm{dL}$ $[23.5 ; 8.5]_{95 \% \mathrm{CI}} p=0.3477$ ) (Fig. 2; ESM Table S4).
In post hoc analyses of the change in $\mathrm{HbA1c}$ from baseline to 26 weeks after IDegAsp initiation, there was a significant improvement in glycaemic control in patients aged $\geq 65$ years (estimated change $-0.2 \%[-0.3 ;-0.1]_{95 \% \mathrm{CI}}$, $p=0.0304)$ that was not observed in those aged $<65$ years (ESM Table S5). There was variation among patients stratified by baseline HbA1c, with a significant increase in HbA1c after 26 weeks of treatment with IDegAsp in those with baseline levels $<7.0 \%$ (estimated change $\left.0.2 \%[0.1 ; 0.3]_{95 \% \mathrm{CI}} p=0.0020\right)$, no significant difference in those with baseline HbA1c $\geq 7.0$ to $<8.0 \%$ and a significant reduction in $\mathrm{HbA1c}$ in those with baseline levels $\geq \quad 8.0 \% \quad$ (estimated change $-0.3 \%$ $\left.[-0.5 ;-0.1]_{95 \% \text { CI }} p=0.0051\right)($ ESM Table S5).

\section{Insulin Dosing}

There were significant reductions in daily insulin dose for both the basal and total (basal and prandial) components after 26 weeks of treatment with IDegAsp (Fig. 3). Observed mean \pm SD basal insulin dose was $15.2 \pm 9.6$ units/day at baseline, decreasing to $11.9 \pm 7.0$ units/day at EOS (estimated change -3.4 units/day $[-3.8 ;-3.0]_{95 \%}$ CI,$\left.p<0.0001\right)$. For total insulin dose, the observed mean \pm SD dose was $18.9 \pm 14.4$ units/day at baseline, decreasing to $18.0 \pm 11.7$ units/day after 26 weeks of treatment with IDegAsp (estimated change -1.0 units/day $[-1.9 ;-0.1]_{95 \%} \quad \mathrm{CI}$, $p=0.0273$ ). Results of the crude analyses were consistent with the main result for basal insulin, but the differences between baseline and EOS were not significant in the crude analysis of the total insulin dose (ESM Table S6). Results of the secondary analyses were consistent with the main results.

At baseline, 49 patients received prandial insulin at an observed mean \pm SD dose of 18.2 \pm 11.6 units/day (Fig. 3). During the study, 30 patients ( $61.2 \%$ of the 49 patients) discontinued prandial insulin. For the 19 patients receiving prandial insulin at EOS, the observed mean \pm SD prandial insulin dose decreased from $20.1 \pm 12.3$ units/day at baseline to $15.7 \pm 11.9$ units/day after 26 weeks; however, 
Table 1 Baseline characteristics of patients enrolled in the study

\section{Baseline characteristic}

Age, years

Male, $n(\%)$

Duration of type 2 diabetes, years

BMI, $\mathrm{kg} / \mathrm{m}^{2}$

HbA1c, \%

FPG, mg/dL

Baseline use of non-insulin diabetes medication, $n$ (\%)

Biguanide

Sulphonylurea

Sulphonamide

Alpha-glucosidase inhibitor

Thiazolidinedione

Dipeptidyl peptidase- 4 inhibitor

Sodium-glucose cotransporter-2 inhibitor

Glucagon-like peptide-1 receptor agonist

Other (excluding insulins)

Baseline glargine administered, $n$ (\%)

Glargine U100

Glargine U300

Baseline glargine dose, units/day

Glargine U100

Glargine U300

Prandial insulin dose, units/day

Frequency of prandial insulin administration, $n$ (\%)

Once daily

Twice daily

Three times daily

Reason for switching to IDegAsp, $n$ (\%)

To improve glycaemic control

Unacceptable hypoglycaemia

Patient wants to try an alternative diabetes medication

Patient wants to try an alternative pen device
Full analysis set $(N=236)$

$63.2 \pm 12.3[n=236]$

$136(57.6)[n=236]$

$14.9 \pm 9.3[n=228]$

$25.8 \pm 4.7[n=224]$

$7.7 \pm 1.0[n=236]$

$148.7 \pm 46.9[n=105]$

$[n=173]$

$94(54.3)$

$26(15.0)$

$0(0.0)$

$30(17.3)$

$16(9.2)$

81 (46.8)

$55(31.8)$

18 (10.4)

$30(17.3)$

$[n=236]$

$143(60.6)$

$93(39.4)$

$15.2 \pm 10.3[n=143]$

$15.1 \pm 8.5[n=93]$

$18.2 \pm 11.6[n=49]$

$[n=49]$

$16(6.8)$

$18(7.6)$

$15(6.4)$

$[n=236]$

214 (90.7)

$2(0.8)$

$1(0.4)$

$1(0.4)$ 
Table 1 continued

\section{Baseline characteristic}

Patient requires a high insulin dose and multiple daily injections

High day-to-day blood glucose variability

Eating habits that fit the pharmacokinetic profile of IDegAsp

Other
Full analysis set $(N=236)$

$10(4.2)$

Data are presented as the mean \pm standard deviation (SD) unless otherwise specified

$B M I$ Body mass index, FPG fasting plasma glucose, glargine U100 insulin glargine 100 units/mL, glargine U300 insulin glargine 300 units/mL, HbAlc glycated haemoglobin, IDegAsp insulin degludec/insulin aspart, $N$ total number of patients, $n$ number of patients with observation

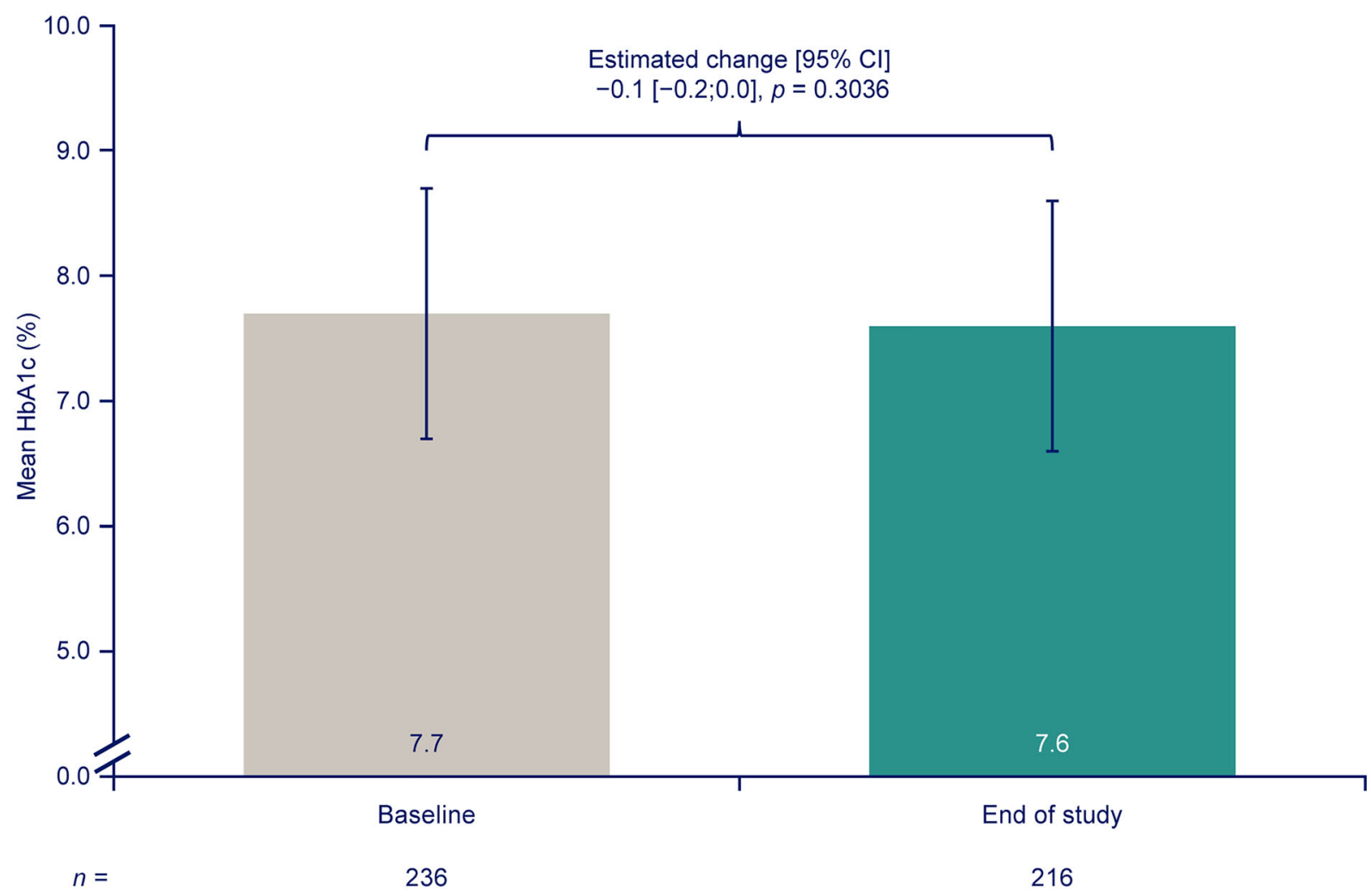

Fig. 1 Change in mean HbAlc from baseline to 26 weeks after insulin degludec/insulin aspart (IDegAsp) initiation. Full analysis set, on-treatment observation period. Data are observed means ( \pm standard deviation [SD]). Estimated mean change (from baseline to end of study) was derived by an adjusted mixed model for repeated measurements (MMRM) with an unstructured covariance matrix, time and time squared as fixed effects, and patient and patient $\times$ time as random coefficients. The model included baseline HbAlc, time, age, sex, diabetes duration, body mass index (BMI), baseline prandial insulin, baseline glucagon-like peptide-1 receptor agonist (GLP-1 RA) and study site as covariates. CI Confidence interval, HbAlc glycated haemoglobin, $n$ number of patients contributing data to time point 


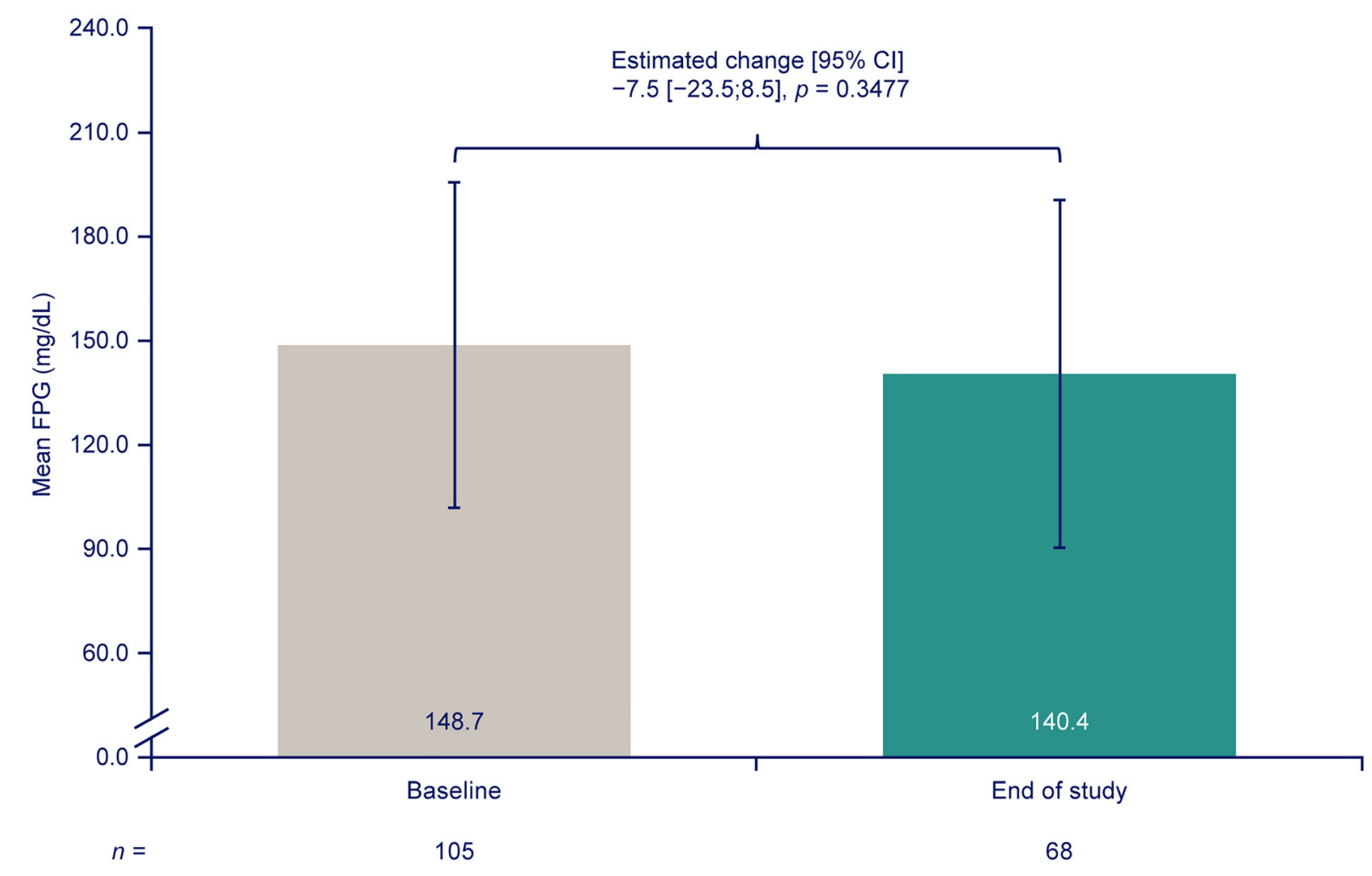

Fig. 2 Change in mean FPG from baseline to 26 weeks after IDegAsp initiation. Full analysis set, on-treatment observation period. Analysed by a crude and an adjusted MMRM with an unstructured covariance matrix, time and time squared as fixed effects, and patient and patient $\times$

time as random coefficients. The model included baseline FPG, time, age, sex, diabetes duration, BMI, baseline prandial insulin, baseline GLP-1 RA, baseline sulphonylurea/glinides and study site as covariates. FPG Fasting plasma glucose; see Fig. 1 caption for other abbreviations

due to the small patient number, the significance could not be tested.

\section{DTR-QoL Scores}

The total DTR-QoL score improved from an observed mean \pm SD of $67.3 \pm 16.3$ at baseline to $70.6 \pm 16.1$ at EOS (estimated change 3.4 $[1.3 ; 5.4]_{95 \%} \quad$ CI $\left.\quad p=0.0012\right)$. There were numerical improvements in median score for all domains between baseline and 26 weeks after initiating IDegAsp, with only one exceptionhypoglycaemia-where the score stayed the same (Fig. 4). The 'anxiety and dissatisfaction with treatment' score increased from an observed median of 56.3 at baseline to 62.5 at EOS; the 'social and daily activities' score improved from 75.6 to 82.1 ; and the

'satisfaction with treatment' score improved from 50.0 to 54.2 ; in contrast, the 'hypoglycaemia' score did not change (83.3 at baseline and EOS). In analyses stratified by baseline HbA1c, there were significant improvements in total DTR-QoL score after switching to IDegAsp in patients with baseline HbA1c $\geq 7.0$ to $<8.0 \%$ or $\geq 8.0 \%$ ( $p<0.05$ for both), but no significant difference in those with baseline HbA1c $<7.0 \%$ (ESM Table S7).

\section{Treatment Preference}

Overall, 186 patients (78.8\%) reported that they were willing to continue treatment with IDegAsp at EOS, and 175 (74.2\%) preferred IDegAsp to their previous treatment (ESM Fig. S3). 


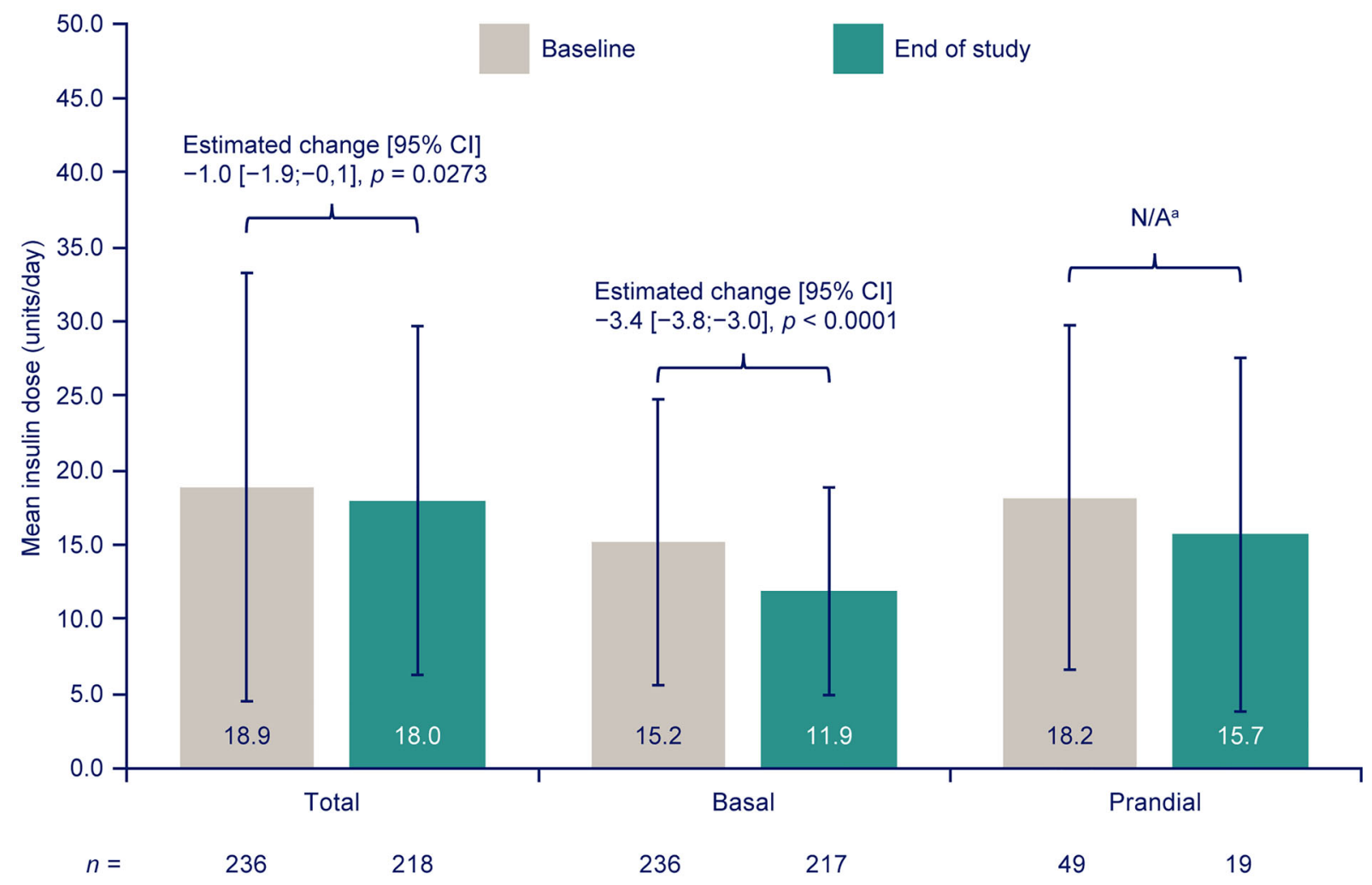

Fig. 3 Change in mean insulin dose from baseline to 26 weeks after IDegAsp initiation. Full analysis set, ontreatment observation period. Data are observed means ( \pm SD). Estimated mean change (from baseline to end of study) was derived using adjusted MMRMs with an unstructured covariance matrix, time and time squared as fixed effects, and patient and patient $\times$ time as random

coefficients. The models included baseline insulin dose, time, age, sex, diabetes duration, BMI, baseline prandial insulin, baseline GLP-1 RA, baseline sulphonylurea/glinides and study site as covariates. Superscript 'a' indicates that insufficient data were available for statistical analysis. $N / A$ Not applicable; see Fig. 1 caption for other abbreviations

\section{Hypoglycaemia}

The observed incidence rate of both overall and nocturnal non-severe hypoglycaemia decreased between the 4-week period prior to initiating IDegAsp and the 4-week period before EOS (Table 2). Prior to initiating IDegAsp, the rate of overall non-severe hypoglycaemia was 3.86 events per patient-year, compared with 2.44 events per patient-year after initiating IDegAsp (estimated rate ratio 0.63 [0.29; $1.38]_{95 \% \text { CI }} p=0.2496$ ). Similarly, there were 1.02 and 0.31 events per patient-year of nocturnal non-severe hypoglycaemia in the periods before and after initiating IDegAsp, respectively

(estimated rate ratio $0.31[0.07 ; 1.37]_{95 \%}$ CI, $p=0.1212$ ). However, the numerical trends toward lower rates of non-severe hypoglycaemia after switching to IDegAsp were not statistically significant. There was a single event of severe hypoglycaemia within either of the 26-week periods before and after initiating IDegAsp that took place in one patient before initiating IDegAsp; the low incidence precluded statistical comparisons of severe hypoglycaemia (Table 2). The secondary analyses of non-severe hypoglycaemia rates were consistent with the primary analyses (ESM Table S8). 


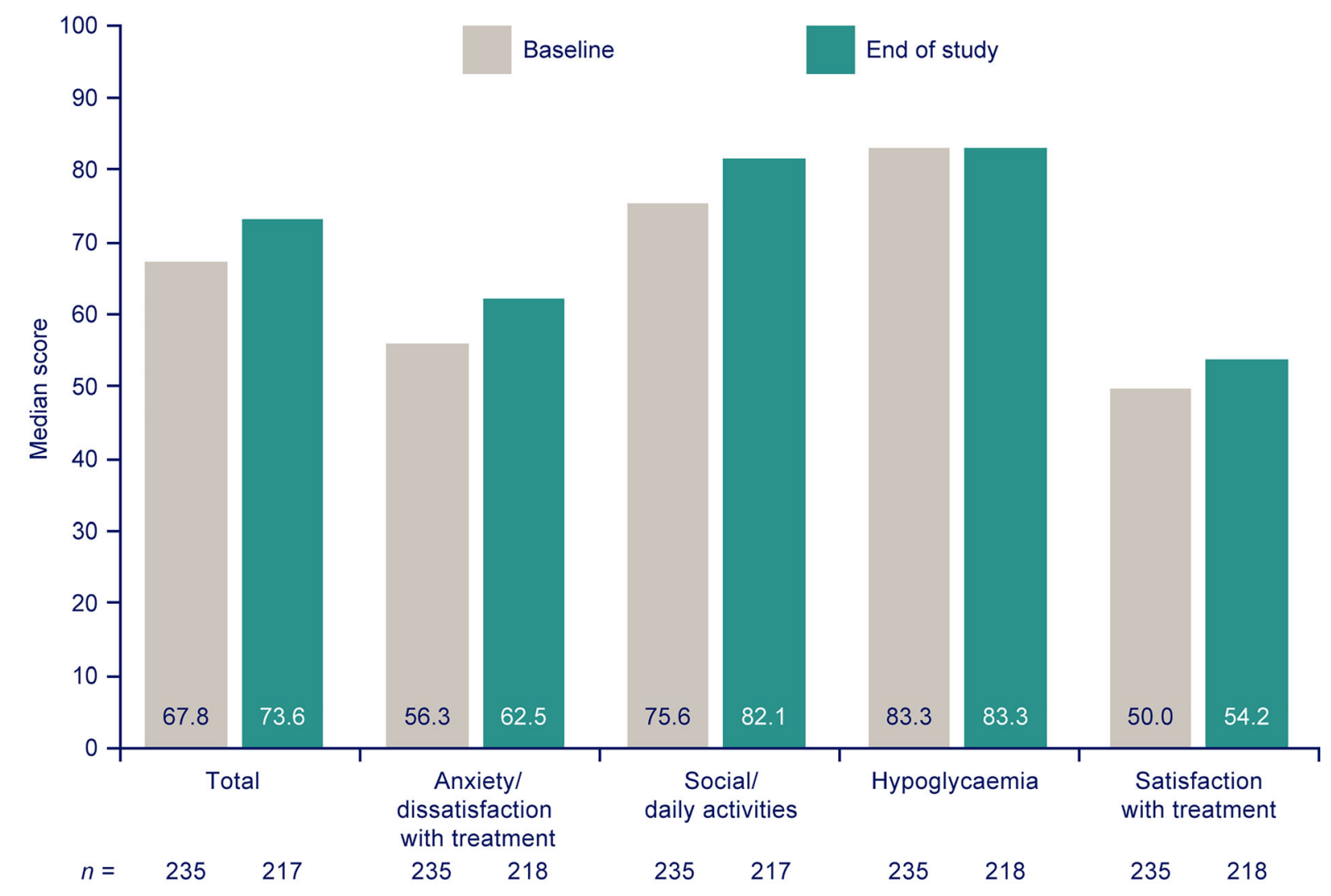

Fig. 4 Median Diabetes Therapy-Related Quality of Life (DTR-QoL) scores at baseline and 26 weeks after IDegAsp initiation. Data are observed median scores. $n$ Number of patients contributing data to time point

\section{Adverse Events}

Overall, 330 AEs were reported in 112 patients (47.5\%) (Table 3). Of the 330 AEs, 316 were non-serious and 311 were judged to be mild in severity. A total of 14 serious AEs (SAEs) were reported in 13 patients, of which nine were deemed to be of mild or moderate severity and five deemed to be severe. All SAEs were determined unlikely to be related to IDegAsp with a single exception: one SAE-'hypoglycaemia'was possibly related to IDegAsp. By system organ class, the most frequently occurring AEs were metabolism and nutritional disorders (21.6\% of patients), infections and infestations (17.4\%) and gastrointestinal disorders (5.9\%). Within these system organ classes, the most frequently occurring AEs were reported for the following preferred terms: hypoglycaemia (19.9\% of patients), nasopharyngitis (10.2\%) and influenza (2.1\%). There were no pregnancies or deaths reported during the study.

\section{DISCUSSION}

In the present real-world, prospective, non-interventional study, Japanese patients with T2D were switched to IDegAsp from glargine U100 or U300, with or without prandial insulin, and as part of routine clinical practice. In this patient population, switching to IDegAsp for 26 weeks was associated with maintenance of glycaemic control, a lower daily total and basal insulin dose requirement, a similar incidence of non-severe hypoglycaemia and improvements in QoL in comparison with baseline prior to the switch.

Glycaemic control was similar at baseline and EOS, although there were numerical trends toward reductions in HbA1c $(-0.1 \% ; p=$ non- 
Table 2 Hypoglycaemia within the periods prior to, and after, initiating IDegAsp

\begin{tabular}{|c|c|c|c|c|c|c|}
\hline \multirow[t]{2}{*}{ Hypoglycaemiaa } & \multicolumn{2}{|c|}{$\begin{array}{l}\text { Events prior to } \\
\text { initiating } \\
\text { IDegAsp }\end{array}$} & \multicolumn{2}{|c|}{$\begin{array}{l}\text { Events after } \\
\text { initiating } \\
\text { IDegAsp }\end{array}$} & \multirow[t]{2}{*}{ Estimated rate ratio $[95 \% \mathrm{CI}]$} & \multirow[t]{2}{*}{$p$ value } \\
\hline & $\bar{n}$ & $R$ & $n$ & $R$ & & \\
\hline Overall non-severe hypoglycaemia & 27 & 3.86 & 21 & 2.44 & $0.63[0.29 ; 1.38]$ & 0.2496 \\
\hline Nocturnal non-severe hypoglycaemia & 9 & 1.02 & 3 & 0.31 & $0.31[0.07 ; 1.37]$ & 0.1212 \\
\hline Overall severe hypoglycaemia & 1 & $\mathrm{~N} / \mathrm{A}^{\mathrm{a}}$ & 0 & $\mathrm{~N} / \mathrm{A}^{\mathrm{a}}$ & $\mathrm{N} / \mathrm{A}^{\mathrm{a}}$ & $\mathrm{N} / \mathrm{A}^{\mathrm{a}}$ \\
\hline Nocturnal severe hypoglycaemia & 0 & $\mathrm{~N} / \mathrm{A}^{\mathrm{a}}$ & 0 & $\mathrm{~N} / \mathrm{A}^{\mathrm{a}}$ & $\mathrm{N} / \mathrm{A}^{\mathrm{a}}$ & $\mathrm{N} / \mathrm{A}^{\mathrm{a}}$ \\
\hline
\end{tabular}

Self-reported hypoglycaemic events in the full analysis set and on-treatment observation period. Estimated rate ratios were based on negative binomial regression models, with log-transformed follow-up time as offset, and compared the incidence of non-severe hypoglycaemia within the 4-week period prior to initiating IDegAsp and the 4-week period prior to end of study or discontinuation. Severe hypoglycaemia was reported for the 26-week period prior to initiating IDegAsp and the 26-week period prior to end of study or discontinuation. Non-severe hypoglycaemia was defined as an event with symptoms and/or a self-monitored blood glucose value $\leq 70 \mathrm{mg} / \mathrm{dL}$. Severe hypoglycaemia was defined as an event requiring assistance from another person to actively administer carbohydrate, glucagon, or take another corrective action. Nocturnal events were categorised based on the patient's answer to the question, 'how many of these occurred between midnight and early morning'

$C I$ confidence interval, $n$ number of patients with an event, $N / A$ not applicable, $R$ events per patient-year

${ }^{a}$ Insufficient data for statistical analysis

significant) and FPG $(-7.5 \mathrm{mg} / \mathrm{dL} ; p=$ nonsignificant). While the differences in glycaemic control after switching from glargine U100 or U300 to IDegAsp were not statistically significant in this real-world setting, there were statistically significant FPG improvements with IDegAsp versus BIAsp 30 in two treat-to-target RCTs of insulin-experienced patients with T2D from Japan [16, 17]. Moreover, once-daily IDegAsp was superior to once-daily glargine U100 with respect to the change from baseline to 26 weeks in HbA1c (estimated treatment difference $-0.28 \%[-0.46 ;-0.10]_{95 \% \text { CI }} p<0.01$ ) in a treat-to-target RCT of insulin-naïve Japanese patients with T2D [15]. There are key differences between RCTs and real-world studies, with clincal trials characterised by a tendency toward strict eligibility criteria, close monitoring, treat-to-target designs, and a fixed-treatment regimen [18]. Of note, the patient populations enrolled in the RCTs of IDegAsp in Japan had higher baseline HbA1c levels (8.3-8.4\%) compared with the real-world cohort in the present study (7.7\%).
Elderly patients have been highlighted as an important subgroup to consider and research by the JDS, and the development of therapies that provide glycaemic control without increasing hypoglycaemia is of particular importance in this patient population [24]. In the present study, post hoc analyses indicated that switching to IDegAsp was associated with a significant improvement in $\mathrm{HbA1c}$ in patients aged $\geq 65$ years, which was not observed in those aged $<65$ years, with no episodes of severe hypoglycaemia in patients of any age. Postprandial hyperglycaemia makes a greater contribution to total hyperglycaemia in people aged $\geq 65$ years than in those aged $<65$ years [25]. Given that most patients in this study were not receiving prandial insulin, targeting postprandial glucose in addition to FPG after the switch to IDegAsp may have a greater impact on glycaemic control in the elderly compared with younger people. However, as this study was not powered for subgroup analyses, the results of the post hoc analysis should be interpreted with caution. 
Table 3 Safety outcomes over 26 weeks after initiating IDegAsp

\begin{tabular}{|c|c|c|c|}
\hline Adverse event & $n$ & $\%$ & $\boldsymbol{E}$ \\
\hline Any & 112 & 47.5 & 330 \\
\hline \multicolumn{4}{|l|}{ Severity } \\
\hline Mild & 101 & 42.8 & 311 \\
\hline Moderate & 12 & 5.1 & 13 \\
\hline Severe & 6 & 2.5 & 6 \\
\hline \multicolumn{4}{|l|}{ Seriousness } \\
\hline Non-serious & 104 & 44.1 & 316 \\
\hline Serious & 13 & 5.5 & 14 \\
\hline \multicolumn{4}{|l|}{ Relatedness to IDegAsp } \\
\hline Probable & 29 & 12.3 & 106 \\
\hline Possible & 25 & 10.6 & 80 \\
\hline Unlikely & 83 & 35.2 & 144 \\
\hline \multicolumn{4}{|l|}{ Outcome } \\
\hline Recovered/resolved & 96 & 40.7 & 286 \\
\hline Recovering/resolving & 9 & 3.8 & 10 \\
\hline Recovered/resolved with sequelae & 0 & 0 & 0 \\
\hline Not recovered/not resolved & 28 & 11.9 & 33 \\
\hline Fatal & 0 & 0 & 0 \\
\hline Unknown & 1 & 0.4 & 1 \\
\hline \multicolumn{4}{|c|}{ Observed in $\geq 5 \%$ of patients, by SOC and PT } \\
\hline Metabolism and nutrition disorders & 51 & 21.6 & 195 \\
\hline Hypoglycaemia & 47 & 19.9 & 190 \\
\hline Infections and infestations & 41 & 17.4 & 51 \\
\hline Nasopharyngitis & 24 & 10.2 & 27 \\
\hline Gastrointestinal disorders & 14 & 5.9 & 19 \\
\hline
\end{tabular}

Observed events in the full analysis set

$\%$ Proportion of patients with an event, $E$ number of events, $n$ number of patients with an event, $P T$ preferred term, $S O C$ system organ class

Over 26 weeks, switching to IDegAsp from glargine U100 or U300 was associated with significant reductions in the daily total and basal insulin dose; furthermore, numerically fewer patients required prandial insulin injections at EOS compared with baseline, with a numerical trend toward a lower daily prandial insulin dose after initiating IDegAsp. As most patients were not receiving prandial insulin at baseline, the basal insulin dose reduction at EOS is likely to reflect the additional contribution of the prandial component of IDegAsp. The reduction in total insulin dose, trend towards lower prandial insulin dose and reduction in the number of prandial insulin injections, albeit in a small number of patients in this study, may have implications in clinical practice, such as fewer AEs and lower healthcare costs. Additionally, the fewer injections when compared with basal-bolus insulin regimens may help to address some of the barriers to insulin intensification and reduce clinical inertia.

In a small, prospective, observational study published in 2018, there was a significant QoL improvement in patients with T2D from Japan who had switched from twice-daily pre-mixed insulin therapy requiring resuspension (BIAsp 30 or biphasic human insulin) to twicedaily IDegAsp [26]. In the present study, switching from glargine with or without prandial insulin to once-daily IDegAsp (95.3\% switched to once-daily dosing) was associated with a significant improvement in total DTR-QoL score, which appeared to be numerically consistent across most domains. In the post hoc analyses, improvements in total DTR-QoL score were observed in patients with baseline HbA1c $\geq 7.0$ to $<8.0 \%$ or $\geq 8.0 \%$ ( $p<0.05$ for both), but there was no significant difference in those with baseline HbA1c $<7.0 \%$. These results align somewhat with those of the post hoc analysis of the primary endpoint-the change in HbA1c from baseline to EOS-where improvements in glycaemic control were observed in patients with baseline $\mathrm{HbA} 1 \mathrm{c} \geq 8.0 \%$ but there was no difference in those with baseline HbA1c $\geq 7.0$ to $<8.0 \%$, and a significant increase in HbA1c in those with baseline levels $<7.0 \%$. Taken together, these post hoc findings suggest that patients with $\mathrm{HbA} 1 \mathrm{c} \geq 8.0 \%$ at baseline derived the greatest benefits from the treatment switch from glargine U100 or U300 to IDegAsp in terms of improved glycaemic control and QoL. Improvements in QoL with IDegAsp may be an 
important driver of the reported preference for IDegAsp compared with glargine by most patients, as well as for patients' stated willingness to continue IDegAsp treatment after the study.

During the study, there were trends toward a reduction in overall and nocturnal non-severe hypoglycaemia, which did not reach statistical significance for either endpoint. In general, treat-to-target RCTs have found similar rates of overall and nocturnal confirmed hypoglycaemia with IDegAsp versus insulin comparators in both insulin-experienced and insulinnaïve Japanese patients [15-17]. However, the rate of nocturnal confirmed hypoglycaemia was found to be significantly lower with twice-daily IDegAsp versus twice-daily BIAsp 30 in insulinexperienced Japanese patients with T2D in a subgroup analysis of a pan-Asian RCT [17].

In the present study, the incidence of severe hypoglycaemia could not be statistically assessed, as there were too few events. However, this low incidence of severe hypoglycaemia may be a positive indication for the use of IDegAsp in Japanese patients with T2D. Fear of hypoglycaemia is a barrier to insulin initiation and intensification [27], particularly among elderly patients with diabetes who tend to be susceptible to severe hypoglycaemia and who are increasing in numbers in Japan [24]. In our study, switching to IDegAsp was generally well tolerated, with only 19 patients discontinuing treatment over the study period. Additionally, the reported AEs were consistent with the known safety profile of IDegAsp in Japanese patients with T2D $[15,16]$, and no new safety or tolerability signals emerged. Of the SAEs reported, only one was determined to be possibly related to IDegAsp treatment.

Our findings provide important insights into the use of IDegAsp in a real-world setting in Japan and have the potential to be generalised across the broad Japanese population due to the real-world, multicentre design of the study. Few study inclusion and exclusion criteria were utilised to better reflect the general adult Japanese population with T2D. Compared with a previous real-world study in Japanese patients [26], this study enrolled a larger cohort and there were high rates of study completion, indicating a robust dataset. However, there are some key limitations that should be considered when interpreting the results of this study. First, although data were collected prospectively, due to the open-label, non-interventional study design, there is the potential for reporting bias and confounding. Secondly, as this was a singlearm study with no comparator group, the contribution of a placebo effect cannot be ruled out, and any other additional influences that resulted in changes in endpoints from baseline could not be investigated.

\section{CONCLUSION}

This was a real-world, prospective, non-interventional study of Japanese patients with T2D who switched to IDegAsp from glargine U100 or U300, with or without prandial insulin, as part of routine clinical practice. Our findings suggest that, in this patient population, switching to IDegAsp for 26 weeks is associated with maintenance of glycaemic control, a lower total and basal insulin dose requirement, a similar incidence of non-severe hypoglycaemia and improvements in QoL, in comparison with baseline prior to the switch. Post hoc analyses indicated that switching to IDegAsp was associated with improved glycaemic control in patients aged $\geq 65$ years and in those with baseline HbA1c $\geq 8.0 \%$.

\section{ACKNOWLEDGEMENTS}

We thank all participants of this study.

Funding. Sponsorship for this study and the journal's Rapid Service Fee were funded by Novo Nordisk A/S and Novo Nordisk Pharma Ltd.

Medical Writing and Editorial Assistance. Medical writing and editorial assistance for the development of this manuscript, under the direction of the authors, was provided by Chloe Harrison and Helen Marshall, of Ashfield MedComms, an Ashfield Health company, and funded by Novo Nordisk Pharma Ltd. 
Authorship. All named authors meet the International Committee of Medical Journal Editors (ICMJE) criteria for authorship for this article, take responsibility for the integrity of the work and have given their approval for this version to be published.

Author Contributions. Takahisa Hirose was the principal physician of the study. Helene Nordahl was the responsible statistician. All authors had access to the study data, contributed to data interpretation, reviewed and contributed to the content of the manuscript and approved the manuscript for publication.

List of Investigators. Hiroshi Ohashi; Yuri Ono; Futoshi Ebara; Shuichi Fukuda; Takahisa Hirose; Dai Shimono; Daigaku Uchida; Fuminobu Okuguchi; Akira Yamauchi; Takashi Sasaki; Shinichiro Shirabe; Hisamoto Kuroda; Mitsuo Imura; Hirotaka Watada; Hideto Ishii; Hiroaki Seino; Yukari Shimizu; Yukiko Onishi; Toshiyuki Sugiura; Koji Sakai; Masaki Owari; Kiyoshi Takekawa; Hideki Nishimura; Ai Sato; Masako Waki; Tetsuya Enomoto; Ryo Suzuki; Tomotada Odaka; Kuninobu Takai.

Disclosures. Lei Liu and Helene Nordahl are employees of Novo Nordisk A/S. Yuiko Yamamoto is an employee of Novo Nordisk Pharma Ltd. Fumika Shigiyama declares no conflicts of interest. Ryo Suzuki has received consulting fees and/or speakers' bureau fees from Eli Lilly Japan K.K., Sanofi K.K. and Novo Nordisk Pharma Ltd. Takahisa Hirose has received research funds from AstraZeneca K.K., Boehringer Ingelheim Pharmaceuticals, Inc., Mitsubishi Tanabe Pharma Corporation, Astellas Pharma Inc., Taisho Pharmaceutical Co., Ltd., Takeda Pharmaceutical Co., Ltd., Ono Pharmaceutical Co., Ltd., Eli Lilly Japan K.K., MSD K.K., Daiichi Sankyo Co., Ltd., Sumitomo Dainippon Pharma Co., Ltd., Novo Nordisk Pharma Ltd., Nippon Boehringer Ingelheim Co., Ltd., Sanofi K.K., Bayer Yakuhin, Ltd., Soiken Inc., Kissei Pharmaceutical Co., Ltd. and Novartis Pharma K.K.; and received lecture fees from Sanofi K.K., Eli Lilly Japan K.K., Novo Nordisk Pharma Ltd., Takeda Pharmaceutical Co., Ltd., MSD K.K., Sumitomo Dainippon Pharma Co., Ltd.,
Novartis Pharma K.K., Nippon Boehringer Ingelheim Co., Ltd., Ono Pharmaceutical Co., Ltd., Daiichi Sankyo Co., Ltd., Mitsubishi Tanabe Pharma Corporation, Kissei Pharmaceutical Co., Ltd. and AstraZeneca K.K.

Compliance with Ethics Guidelines. The study was conducted in accordance with the Declaration of Helsinki, the Guidelines for Good Pharmacoepidemiology Practice, and the Guideline on Good Pharmacovigilance Practices (Module VI). Additionally, the Ethical Guidelines for Medical and Health Research Involving Human Subjects were followed, as required in Japan. The study protocol was approved by the ethics committee of Toho University Omori Medical Center, Tokyo, Japan, and by the appropriate independent ethics boards/institutional review boards at each site. A full list of the research ethics boards/institutional review boards, with their reference numbers, is provided in ESM Table S1. All patients provided written informed consent prior to participation.

Data Availability. The datasets generated during and/or analysed during the current study are available from the corresponding author on reasonable request.

Open Access. This article is licensed under a Creative Commons Attribution-NonCommercial 4.0 International License, which permits any non-commercial use, sharing, adaptation, distribution and reproduction in any medium or format, as long as you give appropriate credit to the original author(s) and the source, provide a link to the Creative Commons licence, and indicate if changes were made. The images or other third party material in this article are included in the article's Creative Commons licence, unless indicated otherwise in a credit line to the material. If material is not included in the article's Creative Commons licence and your intended use is not permitted by statutory regulation or exceeds the permitted use, you will need to obtain permission directly from the copyright holder. To view a copy of this licence, visit http://creativecommons.org/licenses/by$\mathrm{nc} / 4.0 /$. 


\section{REFERENCES}

1. International Diabetes Federation (IDF). IDF diabetes atlas, 9th edn. Brussels: IDF. 2019. https:// www.diabetesatlas.org/en/resources/.

2. Charvat H, Goto A, Goto M, et al. Impact of population aging on trends in diabetes prevalence: a meta-regression analysis of 160,000 Japanese adults. J Diabetes Investig. 2015;6:533-42.

3. Araki E, Goto A, Kondo T, et al. Japanese clinical practice guideline for diabetes 2019. J Diabetes Investig. 2020;11:1020-76.

4. Morimoto A, Tatsumi Y, Deura K, et al. Impact of impaired insulin secretion and insulin resistance on the incidence of type 2 diabetes mellitus in a Japanese population: the Saku study. Diabetologia. 2013;56:1671-9.

5. Fukushima M, Suzuki H, Seino Y. Insulin secretion capacity in the development from normal glucose tolerance to type 2 diabetes. Diabetes Res Clin Pract. 2004;66(Suppl 1):S37-43.

6. Koga M, Toyomaki A, Miyazaki A, et al. Mediators of the effects of rice intake on health in individuals consuming a traditional Japanese diet centered on rice. PLoS One. 2017;12:e0185816.

7. Home P, Riddle M, Cefalu WT, et al. Insulin therapy in people with type 2 diabetes: opportunities and challenges? Diabetes Care. 2014;37:1499-508.

8. Strain WD, Blüher M, Paldánius P. Clinical inertia in individualising care for diabetes: is there time to do more in type 2 diabetes? Diabetes Ther. 2014;5: 347-54.

9. European Medicines Agency. Ryzodeg (insulin degludec/insulin aspart). Summary of product characteristics. 2020. https://www.ema.europa.eu/ en/documents/product-information/ryzodeg-eparproduct-information_en.pdf. Accessed Jan 2021.

10. Atkin S, Javed Z, Fulcher G. Insulin degludec and insulin aspart: novel insulins for the management of diabetes mellitus. Ther Adv Chronic Dis. 2015;6: 375-88.

11. Kumar A, Franek E, Wise J, Niemeyer M, Mersebach $\mathrm{H}$, Simo R. Efficacy and safety of once-daily insulin degludec/insulin aspart versus insulin glargine (U100) for 52 weeks in insulin-naive patients with type 2 diabetes: a randomized controlled trial. PLoS One. 2016;11:e0163350.

12. Kumar S, Jang HC, Demirag NG, Skjoth TV, Endahl L, Bode B. Efficacy and safety of once-daily insulin degludec/insulin aspart compared with once-daily insulin glargine in participants with Type 2 diabetes: a randomized, treat-to-target study. Diabet Med. 2017;34:180-8.

13. Philis-Tsimikas A, Astamirova K, Gupta Y, et al. Similar glycaemic control with less nocturnal hypoglycaemia in a 38-week trial comparing the IDegAsp co-formulation with insulin glargine U100 and insulin aspart in basal insulin-treated subjects with type 2 diabetes mellitus. Diabetes Res Clin Pract. 2019;147:157-65.

14. Fulcher GR, Christiansen JS, Bantwal G, et al. Comparison of insulin degludec/insulin aspart and biphasic insulin aspart 30 in uncontrolled, insulintreated type 2 diabetes: a phase 3a, randomized, treat-to-target trial. Diabetes Care. 2014;37: 2084-90.

15. Onishi Y, Ono Y, Rabol R, Endahl L, Nakamura S. Superior glycaemic control with once-daily insulin degludec/insulin aspart versus insulin glargine in Japanese adults with type 2 diabetes inadequately controlled with oral drugs: a randomized, controlled phase 3 trial. Diabetes Obes Metab. 2013;15: 826-32.

16. Onishi Y, Yamada K, Zacho J, Ekelund J, Iwamoto Y. Insulin degludec/insulin aspart vs biphasic insulin aspart 30 twice daily in Japanese patients with type 2 diabetes: a randomized controlled trial. J Diabetes Investig. 2017;8:210-7.

17. Taneda S, Hyllested-Winge J, Gall MA, Kaneko S, Hirao K. Insulin degludec/insulin aspart versus biphasic insulin aspart 30 twice daily in insulinexperienced Japanese subjects with uncontrolled type 2 diabetes: subgroup analysis of a Pan-Asian, treat-to-target Phase 3 Trial. J Diabetes. 2017;9: 243-7.

18. Cohen AT, Goto S, Schreiber K, Torp-Pedersen C. Why do we need observational studies of everyday patients in the real-life setting? Eur Heart J Suppl. 2015;17:D2-8.

19. World Medical Association. World Medical Association Declaration of Helsinki: ethical principles for medical research involving human subjects. JAMA. 2013;310:2191-4.

20. Public Policy Committee ISoP. Guidelines for good pharmacoepidemiology practice (GPP). Pharmacoepidemiol Drug Saf. 2016;25:2-10.

21. European Medicines Agency and Heads of Medicines Agencies. Guideline on good pharmacovigilance practices (GVP). Module VI - collection, management and submission of reports of suspected adverse reactions to medicinal products (Rev 2). 2017. https://www.ema.europa.eu/en/documen ts/regulatory-procedural-guideline/guideline-good- 
pharmacovigilance-practices-gvp-module-vicollection-management-submission-reports_en. pdf. Accessed Jan 2021.

22. Ogasawara K. 8. Revised "ethical guidelines for medical and health research involving human subjects." Nihon Hoshasen Gijutsu Gakkai Zasshi. 2017;73:397-402.

23. Seaquist ER, Anderson J, Childs B, et al. Hypoglycemia and diabetes: a report of a workgroup of the American Diabetes Association and the Endocrine Society. J Clin Endocrinol Metab. 2013;98: 1845-59.

24. Japan Diabetes Society. Committee Report: glycemic targets for elderly patients with diabetes: Japan Diabetes Society (JDS)/Japan Geriatrics Society (JGS) Joint Committee on improving care for elderly patients with diabetes. J Diabetes Investig. 2017;8:126-8.

25. Munshi MN, Pandya N, Umpierrez GE, DiGenio A, Zhou R, Riddle MC. Contributions of basal and prandial hyperglycemia to total hyperglycemia in older and younger adults with type 2 diabetes mellitus. J Am Geriatr Soc. 2013;61:535-41.

26. Fujimoto K, Iwakura T, Aburaya M, Matsuoka N. Twice-daily insulin degludec/insulin aspart effectively improved morning and evening glucose levels and quality of life in patients previously treated with premixed insulin: an observational study. Diabetol Metab Syndr. 2018;10:64.

27. Russell-Jones D, Pouwer F, Khunti K. Identification of barriers to insulin therapy and approaches to overcoming them. Diabetes Obes Metab. 2018;20: 488-96. 